\title{
BMJ Open Single assessment of delirium severity during postacute intensive care of chronically critically ill patients and its associated factors: post hoc analysis of a prospective cohort study in Germany
}

\author{
Gloria-Beatrice Wintermann (D) , ${ }^{1}$ Kerstin Weidner, ${ }^{1}$ Bernhard Strauss, ${ }^{2}$ \\ Jenny Rosendahl (i) 2,3
}

To cite: Wintermann G-B, Weidner K, Strauss B, et al. Single assessment of delirium severity during postacute intensive care of chronically critically ill patients and its associated factors: post hoc analysis of a prospective cohort study in Germany. BMJ Open 2020;10:e035733. doi:10.1136/ bmjopen-2019-035733

- Prepublication history and additional material for this paper are available online. To view these files, please visit the journal online (http://dx.doi. org/10.1136/bmjopen-2019035733).

Received 14 November 2019 Revised 23 June 2020 Accepted 02 July 2020

D) Check for updates

(c) Author(s) (or their employer(s)) 2020. Re-use permitted under CC BY-NC. No commercial re-use. See rights and permissions. Published by BMJ.

For numbered affiliations see end of article.

\section{Correspondence to}

Dr Gloria-Beatrice Wintermann; gloria.wintermann@uniklinikumdresden.de

\section{ABSTRACT}

Objectives To assess the delirium severity (DS), its risk factors and association with adverse patient outcomes in chronically critically ill (CCl) patients.

Design A prospective cohort study.

Setting A tertiary care hospital with postacute intensive care units (ICUs) in Germany.

Participants $\mathrm{N}=267 \mathrm{CCl}$ patients with critical illness polyneuropathy and/or critical illness myopathy, aged 18-75 years, who had undergone elective tracheotomy for weaning failure.

Interventions None.

Measures Primary outcomes: DS was assessed using the Confusion Assessment Method for the Intensive Care Unit-7 delirium severity score, within 4 weeks (t1) after the transfer to a tertiary care hospital. In post hoc analyses, univariate linear regressions were employed, examining the relationship of DS with clinical, sociodemographic and psychological variables. Secondary outcomes: additionally, correlations of DS with fatigue (using the Multidimensional Fatigue Inventory-20), quality of life (using the Euro-Quality of Life) and institutionalisation/mortality at 3 (t2) and 6 (t3) months follow-up were computed.

Results Of the $\mathrm{N}=267$ patients analysed, $9.4 \%$ showed severe or most severe delirium symptoms. $4.1 \%$ had a full-syndromal delirium. DS was significantly associated with the severity of illness $(p=0.016,95 \% \mathrm{Cl}-0.1$ to -0.3$)$, number of medical comorbidities $(p<0.001,95 \% \mathrm{Cl} .1$ to .3 ) and sepsis $(p<0.001,95 \% \mathrm{Cl} .3$ to 1.0$)$. Patients with a higher DS at postacute ICU (t1), showed a higher mental fatigue at t2 $(p=0.008,95 \% \mathrm{Cl} .13$ to .37) and an increased risk for institutionalisation/mortality $(\mathrm{p}=0.043,95 \% \mathrm{Cl} 1.1$ to $28.9 / \mathrm{p}=0.015,95 \% \mathrm{Cl} 1.5$ to 43.2 ).

Conclusions IIIness severity is positively associated with DS during postacute care in CCI patients. An adequate management of delirium is essential in order to mitigate functional and cognitive long-term sequelae following ICU. Trial registration number DRKS00003386.

\section{INTRODUCTION}

Every 10th patient with protracted treatment in an intensive care unit (ICU), for example, because of sepsis with multiorgan
Strengths and limitations of this study

- We consecutively enrolled a quite homogeneous sample of long-term ventilated chronically critically ill (CCI) patients, using a straightforward timeline and applying a valid delirium assessment tool, the Confusion Assessment Method for the Intensive Care Unit (CAM-ICU).

- For the first time, the CAM-ICU-7 delirium severity score, based on the CAM-ICU, was used and associated with sociodemographic, clinical and psychological risk factors as well as posthospital outcomes (fatigue, quality of life, institutionalisation/mortality) in CCI patients.

- The study was primarily designed to address posttraumatic stress disorder in $\mathrm{CCl}$ patients, therefore the study sample may be biased towards patients without delirium.

- The delirium assessment tool 'CAM-ICU' was not assessed at multiple time points, which could have led to a further underestimation of the delirium rate as well as delirium severity in CCI patients.

dysfunction, is prone to develop a clinical syndrome which is called chronic critical illness (CCI). ${ }^{12}$ Sepsis or other severe infections may precede CCI, leading to major dysfunctions of the central nervous system, non-focal encephalopathy and neurological long-term complications, ${ }^{34}$ among them critical illness polyneuropathy (CIP) or critical illness myopathy (CIM). ${ }^{256}$ These patients are exposed to prolonged mechanical ventilation and ICU lengths of stay, leading to an increased risk to develop significant sequelae, including delirium. ${ }^{7-10}$ Delirium refers to acute brain failures which usually manifest as acute or exacerbating deficits in global cognition, awareness, attention and/or executive functions. It develops quickly and fluctuates over the course of the 
day. ${ }^{11}$ Findings reveal a wide range of occurrence rates, varying across clinical contexts, admitting diagnoses and hospital departments. ${ }^{12}$ The lowest rate could be proven for general hospital inpatients $\left(\mathrm{eg}, 20.7 \%{ }^{13}\right)$, the highest for older ICU patients $\left(82 \%^{14}\right)$, mechanically ventilated $\left(81.7 \%^{15}\right)$ and CCI patients $(33.8 \%$ to $\left.46.5 \%^{1617}\right)$.

With respect to the an adequate delirium assessment in the ICU setting, the Confusion Assessment Method for the Intensive Care Unit (CAM-ICU) has turned out to be a rapid, valid and reliable instrument for use by non-psychiatric clinicians. ${ }^{18} 19$ However, in order to gain more insight into the clinical course and the underlying mechanistic relationships of delirium, the assessment of delirium severity (DS) seems to be more appropriate. ${ }^{20}$ The measurement of symptom severity may help to detect clinically important syndromes that fall on a continuum between no symptoms and a diagnosis of delirium. This would be of prognostic relevance regarding clinical outcomes. $^{2122}$

However, the validity of a CAM-based scoring system has been proven in former studies within general hospital ${ }^{20}$ and ICU, ${ }^{23}$ but not in CCI patients. Likewise, risk factors (eg, PRE-DELIRIC model: age, infection, severity of illness, use of sedatives) have been mainly studied for critically ill but not CCI patients. ${ }^{24-27}$ The assessment of a valid delirium severity score (DSS) and its predictors is of utmost importance, since delirium displays a major risk factor for the development of long-term cognitive and functional disabilities, months to years after ICU treatment. ${ }^{71628}$ As already shown, predominantly for acutely ill patients, delirium could lead to deficiencies in patients rehabilitation outcomes. For instance, it may increase the risk for persistent ventilator dependence, longer stays in ICU, nursing home residence, the progression of former disorders (eg, dementia) as well as higher rehospitalisation and mortality rates. ${ }^{152023-30}$ In particular, delirium within CCI might cause the development of functional and cognitive limitations, even $>3$ years surviving severe sepsis. ${ }^{7-9}$ As a consequence, these patients may require rehospitalisation, institutionalisation, an increased amount of informal caregiving and thus, leading to higher emotional burdens. The latter maybe associated with the evolvement of psychiatric morbidity, such as depression and fatigue, leading to a diminished health-related quality of life, both in patients and their spouses. ${ }^{31-33}$ However, the association of delirium with psychological disorders has not been investigated, in particular after long-term ICU treatment in CCI patients.

The aims of the present study are: first, to assess the occurrence and severity of delirium in CCI patients during their treatment in a postacute ICU. Second, to examine the association of DS with clinical, sociodemographic and psychological risk factors. Third, to investigate whether DS is associated with patient-reported outcomes after hospitalisation, such as fatigue and health-related quality of life. Fourth, to outline whether DS can predict mortality and institutionalisation at follow-up. Findings may further help to efficiently target preventive therapies at high-risk patients.

\section{METHODS \\ Study design}

We conducted a post hoc analysis of a prospective, longitudinal cohort study, originally designed to examine the frequency of acute stress disorder and post-traumatic stress disorder in CCI patients at three measurement points. ${ }^{34}$ First, CCI patients were investigated according to a delirium/DS within 4 weeks following the transfer from acute-care ICU to a centre for weaning from long-term mechanical ventilation ( $\mathrm{t} 1$ ), referred to as postacute ICU. Second, fatigue, health-related quality of life and patient status (dead, institutionalised vs discharged home) were assessed at follow-up via telephone, 3 (t2) and 6 (t3) months post-transfer. Further details on the study design are published elsewhere. ${ }^{34}$

\section{Patient and public involvement}

Patients were not involved in the study design or in the conduct of the study. Representatives of the intensive care sector were involved in both the design and the implementation of the study results. Laymen reports of the present prospective cohort study are publicly available in German.

\section{Participants}

Between January 2011 and February 2013, we consecutively enrolled a quite homogeneous sample of CCI patients who were transferred from adult ICUs (medical, surgical, cardiac, neurosurgical) to a large tertiary care hospital with postacute ICUs for weaning from long-term mechanical ventilation. All patients, included in the present study, had undergone elective tracheotomy for weaning failure.

We consecutively enrolled patients who fulfilled the following inclusion criteria: presence of a diagnosis of critical illness polyneuropathy (CIP, International Classification of Diseases (ICD)-10: G62.80) or critical illness myopathy (CIM, ICD-10: G72.80) with or without sepsis, age between 18 and 75 years, a minimum length of ICU stay of 6 days, mechanical ventilation, sufficient German language skills, informed consent for study participation and presence of a delirium assessment using the CAM-ICU. ${ }^{1835}$

We excluded patients who refused study participation, who were transferred to another hospital or died before recruitment, showed insufficient German language skills, were sensorily impaired (eg, deaf-mute), persistently intubated and/or comatose, had a diagnosis of dementia (ICD-10 F00-F03), rapidly terminal disease states or an infaust prognosis (eg, malignancy). A flow chart of the recruitment process is shown in figure 1 . In the present post hoc analysis, slight differences to the recruitment numbers of the original study ${ }^{34}$ are mainly due to the inclusion of patients with a delirium or positive CAM-ICU assessment.

\section{Measures}

Socioeconomic characteristics (eg, age, family status, education) and medical history (eg, history of harmful 


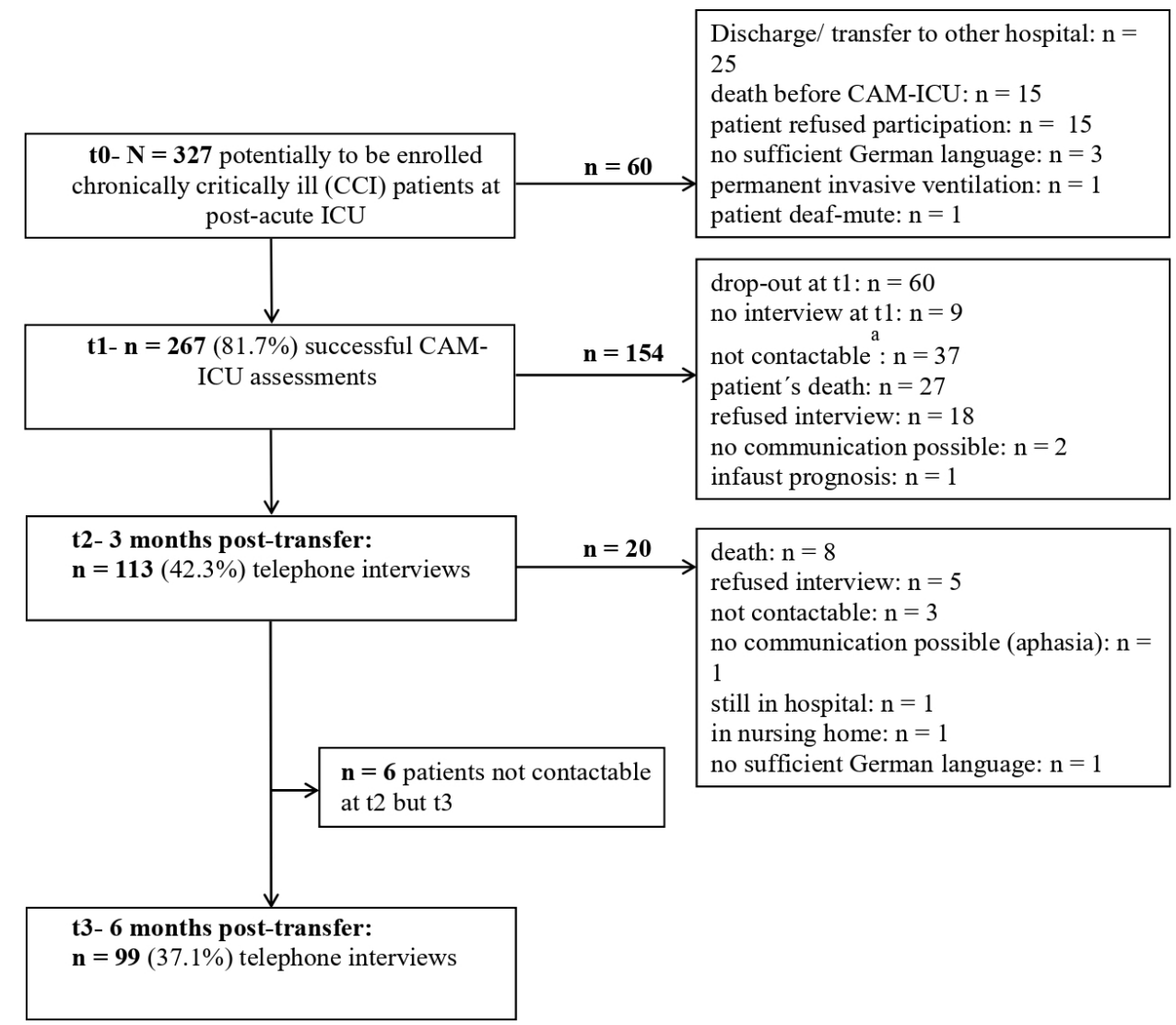

Figure 1 Flow chart displaying the number of potentially to be enrolled chronically critically ill (CCl) patients, the actual number of enrolled patients at $\mathrm{t} 1$ (within 4 weeks after transfer to postacute ICU), at t2 (3 months follow-up) and at $\mathrm{t} 3$ (6 months followup). CAM-ICU, Confusion Assessment Method for the Intensive Care Unit. a: Unknown reason: $n=22$, still in hospital: $n=12$, in nursing home: $n=3$.

alcohol consumption/anxiety or affective disorders, diagnosis of sepsis, number of sepsis episodes, severity of sepsis, site of infection, length of mechanical ventilation from the time of endotracheal intubation to successful unassisted breathing, length of ICU stay, number of medical comorbidities, presence of an organic brain syndrome: ICD-10 F07.9, medication, eg, exposure to antipsychotics, analgetics or sedatives) were extracted from patient record forms at t1. The patients' functional status was assessed by a trained study nurse, using the Barthel index. The Barthel index measures performance in activities of daily living in 11 domains (eg, faecal incontinence, urinary incontinence, help with grooming/toilet use), with values ranging between 0 and 100. A higher value is associated with a better degree of independence from caregivers. Additionally, the early rehabilitation Barthel index was assessed with seven domains (intensive care supervision, tracheostomy tube management and supervision, intermittent or continuous mechanical ventilation, confusion, behavioural disturbances, severe impairment of communication and dysphagia), with a minimum value of -325 and a maximum value of $0 .{ }^{36}$ Both Barthel scales were summed up, yielding scores between -325 and 100. Interrater reliability is very high $(\mathrm{r}=0.95)$. Test-retest reliability is good as well $(\mathrm{r}=0.89) .{ }^{37}$
CAM-ICU: the CAM-ICU ${ }^{18}$ was applied at $\mathrm{t} 1$, within 4 weeks following the transfer from acute-care ICU. It can be used for the non-verbal assessment of a delirium, which is appropriate for mechanically ventilated patients. For this purpose, four domains are evaluated, including (1) an acute onset or fluctuations in the course of mental status, using the Richmond Agitation Sedation Scale (RASS) ${ }^{38}$ at t1; (2) inattention, using the Attention Screening Examination; (3) disorganised thinking, using four simple questions and (4) alteration in the level of consciousness, again using the RASS. The RASS is a 10-level scale ranging from ' -5 ' unarousable to ' +4 ' combative, and shows excellent reliability and acceptable validity in ventilated adult ICU patients. A patient was determined to be delirious if he/she manifested features 1 and 2, plus either feature 3 or 4 . In order to assess DS, we applied a 7-point rating scale (0-7), derived from the CAM-ICU and RASS assessments. Each symptom of delirium is evaluated as described in detail by Khan $e t a l^{23}$ (acute onset and altered level of consciousness: absent (0) or present (1); inattention/disorganised thinking: absent (0), mild (1) and marked (2)). The CAM-ICU-7 DSS is categorised as 0-2 (no delirium), 3-5 (mild to moderate severity) and 6-7 (severe delirium). 
Multidimensional Fatigue Inventory-20 (MFI-20): the MFI-20 $0^{3940}$ is a 20-item self-report measurement of fatigue severity. It covers the five dimensions 'general fatigue', 'physical fatigue', 'mental fatigue', 'reduced motivation' and 'reduced activity'. Items are summed up to a simple total score, with a minimum value of 4 (absence of fatigue) and a maximum value of 20 for each subscale. A total fatigue score is calculated as the sum of the subscale scores (range 20-100). Higher scores indicate higher levels of fatigue. For the present study, particularly the subscale 'mental fatigue' was considered, assessing, for example, the extent of difficulties in concentration, as well as sustaining performance in mental tasks, that might result in transient decline in cognitive performance. The MFI-20 was applied via telephone at 3 (t2) and 6 months (t3) following discharge from acute-care ICU. Validity has been shown for different populations, for example, patients with cancer, army recruits, patients with chronic fatigue syndrome and CCI patients. ${ }^{33}{ }^{39}$ Internal consistency has been reported to be good for the general, physical and mental fatigue dimensions (Cronbach' $\alpha=0.84$ ), and adequate for the subscales reduced activity and reduced motivation (Cronbach's $\alpha>0.65){ }^{39}$

Euro-Quality of Life (EQ-5D-3L): quality of life was assessed with the questionnaire EQ-5D-3L $\mathrm{L}^{41}$ at $\mathrm{t} 2$ and $\mathrm{t} 3$. The EQ-5D-3L measures health-related quality of life on five dimensions (mobility, self-care, usual activities, pain/ discomfort and anxiety/depression), which are evaluated within three severity levels (no problems, some or moderate problems, extreme problems or unable). A single one-dimensional index value is generated based on a simple sum score according to Hinz et $a l_{.}{ }^{42}$

\section{Statistical analysis}

Normal distribution was ascertained by KolmogorovSmirnov test. In case of normally distributed data, arithmetic means and standard deviations (SD), otherwise data medians and interquartile ranges $(\mathrm{IQR})$ are reported. For categorical variables, absolute and relative frequencies are presented. Mann-Whitney U test or t-test was used to compare medians or means of outcome variables between followed up and dropped out patients. Fisher's exact test or $\chi^{2}$ test was run, in case of nominal outcome data. The association of the CAM-ICU-7 DSS with clinical (eg, sepsis, length of mechanical ventilation/ICU stay, severity of medical illness/Barthel index, number of medical comorbidities, medication), sociodemographic (eg, age, gender, family/education status) and psychological variables was analysed using univariate linear regressions. The choice of these variables was mainly driven by research on risk factors for intensive care delirium (see the systematic review by Van Rompaey $e t a t^{24}$ ). We had to adapt our choice of potential predictors to the availability of information on these data in the patient records, mainly because the present study was originally designed to assess the frequency of stress-related disorders in CCI patients. ${ }^{34}$ Since there was only a low percentage of cases with a delirium (see 'Results' section), we did not calculate logistic regression analyses. Exploratory, the CAM-ICU-7 DSS was correlated with the subscales of the MFI-20, the health-related quality of life as assessed with the EQ-5D-3L and the status (dead, institutionalised vs discharged home) after hospital discharge at t2 and t3, using Spearman's rank correlation coefficients. Additionally, the CAM-ICU-7 DSS was entered in a multinomial logistic regression analysis, controlling for covariates as partially suggested by Khan $e t a t^{23}$ (age, gender, length of ICU/mechanical ventilation, number of comorbidities, sepsis, education/family status). Both the CAM-ICU-7 DSS and MFI-20 subscale mental fatigue were correlated with the length of mechanical ventilation/length of ICU stay, using Spearman's rank correlations. For all analyses, a significance level of $\alpha=0.05$ (two-sided) and $\mathrm{z}$ standardised values were applied. All data were analysed using SPSS V.26.0.0.0 (SPSS, Chicago, Illinois, USA).

\section{RESULTS}

\section{Baseline characteristics}

Of $\mathrm{N}=327$ potentially eligible CCI patients, a subsample of $n=267(81.7 \%)$ patients could be successfully evaluated using the CAM-ICU (see figure 1, flow chart). About three-quarter of the patients were male $(72.3 \%)$. Onethird of the sample $(31.8 \%)$ was older than 65 years, with a median age of 61.6 years (range 25.6-71.9 years). More than half of the patients $(58.8 \%)$ reported at least 10 years of education (table 1 ). Median length of ICU stay was 73 days and length of mechanical ventilation 53 days. Nearly three-quarter $(70.4 \%)$ of the total sample had an anamnesis of sepsis, mostly located at the respiratory tract. Most patients suffered from an acute respiratory insufficiency $(80.1 \%)$. One-third had a hypertension $(55.4 \%)$, left heart failure $(33.7 \%)$, atrial fibrillation $(35.2 \%)$ or coronary heart disease $(30.0 \%)$ (see online supplemental table S1). More than half of the patients $(50.9 \%)$ had $>10$ medical comorbidities. Those patients who could not be enrolled (drop outs at t0 or t1) were more severely ill than the patients enrolled in the present study (for further information, see table 1, online supplemental table S2).

\section{Frequency of delirium and delirium severity score}

Inter-rater reliability (without the RASS score) for the CAM-ICU assessment in the present study was calculated, with an intraclass correlation coefficient (ICC) of 0.66 that lies within the range reported for different patients samples $(\mathrm{ICC}=0.64-0.82)^{38} ; 4.1 \% \quad(\mathrm{n}=11)$ of the enrolled sample fulfilled the criteria for a delirium according to the CAM-ICU assessment at t1. The median RASS score corresponded to an alert state of attention (median $=0$, $\mathrm{IQR}=0-0$ ). According to the classification of the CAMICU-7 DSS (median=0, IQR $=0-0), 79 \% \quad(n=211)$ of the patients had no delirium, $11.6 \% \quad(\mathrm{n}=31)$ had a mild to moderate and $9.4 \%(n=25)$ had a severe form.

\section{Risk factors associated with DSS}

As significant risk factors for more severe delirium symptoms at time of recruitment (t1), mostly clinical variables 


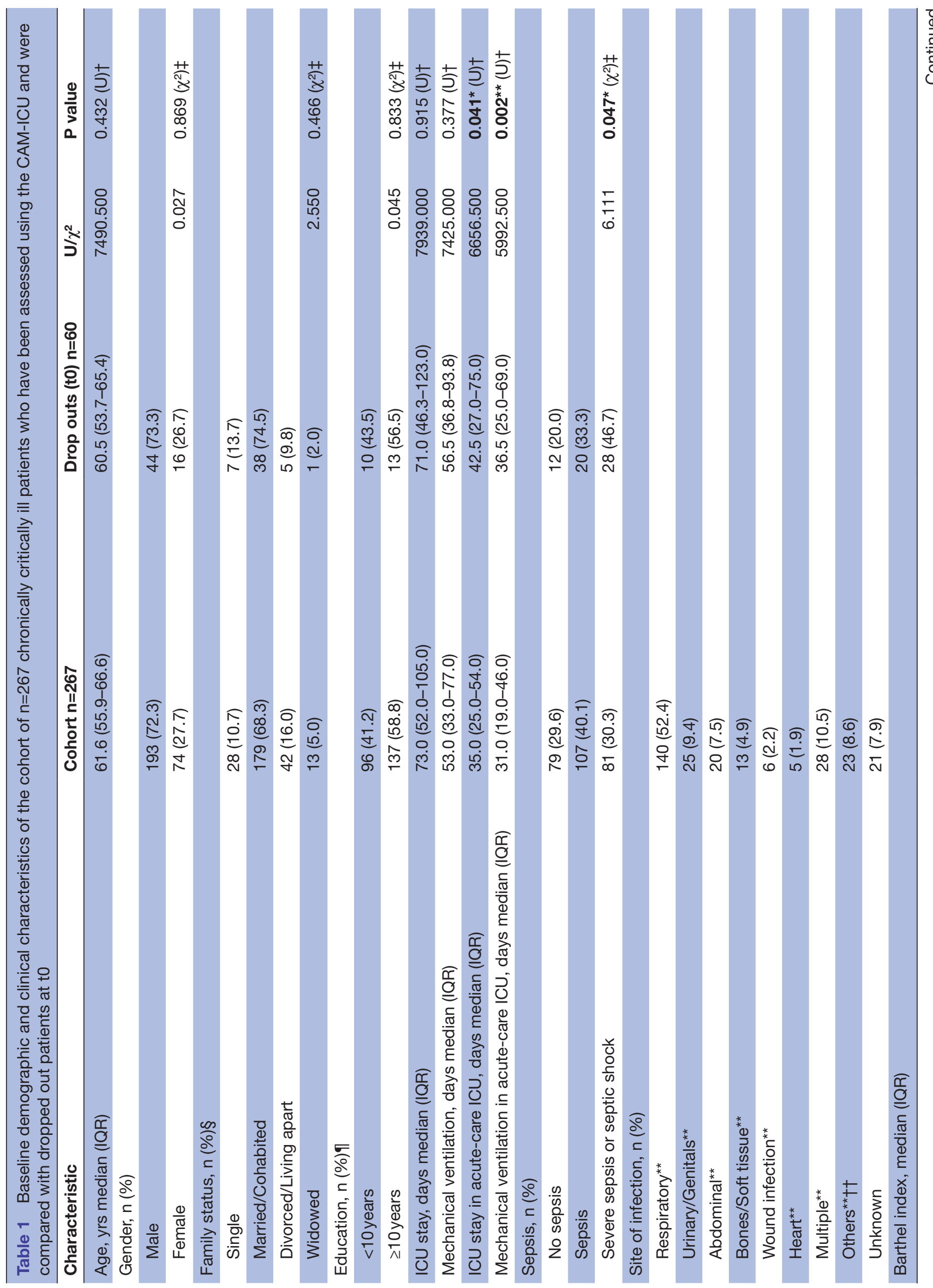




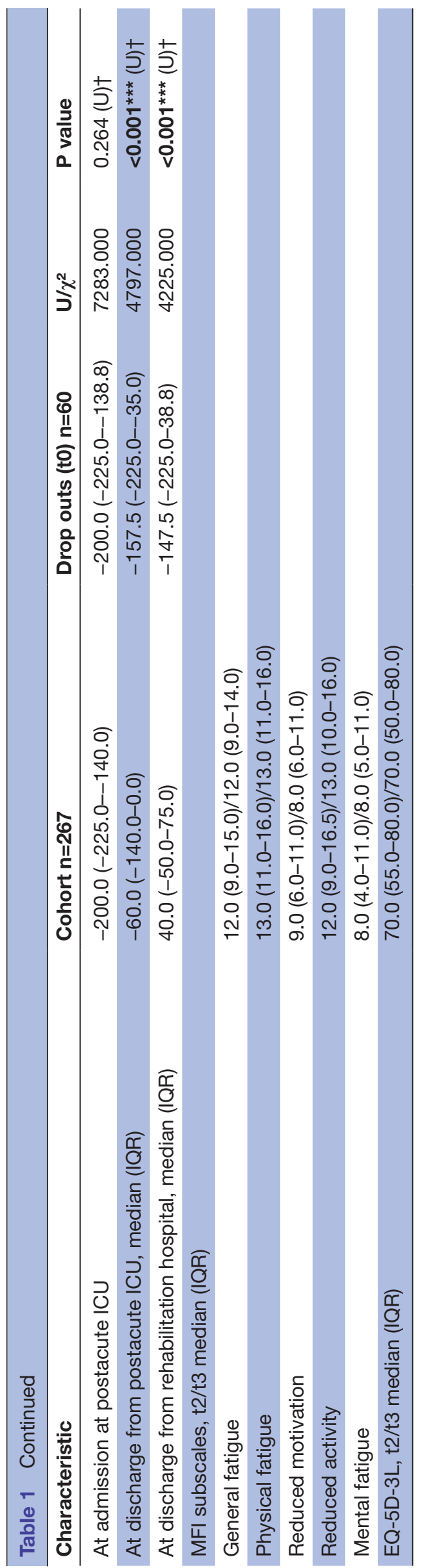

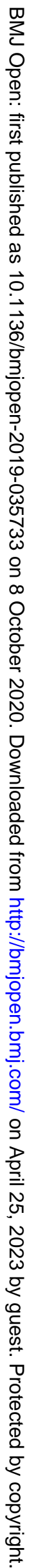


Table 2 Univariate logistic regression using Enter as method for the identification of sociodemographic, clinical and psychological predictors of the CAM-ICU-7 delirium severity score during postacute intensive care in chronically critically ill (CCl) patients $(n=267)$

\begin{tabular}{|c|c|c|c|}
\hline \multicolumn{4}{|l|}{ Univariate linear regression } \\
\hline & Beta (standardised) & $95 \% \mathrm{Cl}$ & $P$ value \\
\hline \multicolumn{4}{|l|}{ Sociodemographic variables } \\
\hline Age & 0.056 & -0.066 to 0.180 & 0.361 \\
\hline Gender & 0.035 & -0.194 to 0.353 & 0.566 \\
\hline Family status, no partnership vs partnership $\dagger$ & -0.003 & -0.267 to 0.255 & 0.964 \\
\hline Education status $<10$ years vs $\geq 10$ years $\ddagger$ & -0.091 & -0.399 to 0.069 & 0.167 \\
\hline \multicolumn{4}{|l|}{ Clinical variables } \\
\hline Number of sepsis episodes & 0.114 & -0.007 to 0.237 & 0.064 \\
\hline Sepsis in postacute ICU & 0.231 & 0.313 to 0.962 & $<0.001^{\star \star \star}$ \\
\hline Barthel index at admission in postacute ICU & -0.147 & -0.271 to 0.028 & $0.016^{*}$ \\
\hline Number of medical comorbidities & 0.221 & 0.105 to 0.344 & $<0.001^{* * *}$ \\
\hline Use of antipsychotic medication (in postacute ICU) & 0.046 & -0.191 to 0.423 & 0.459 \\
\hline Use of sedative medication (in postacute ICU) & -0.023 & -0.311 to 0.210 & 0.702 \\
\hline Use of analgetic medication (in postacute ICU) & 0.030 & -0.204 to 0.341 & 0.622 \\
\hline Organic brain syndrome (in acute-care ICU) & 0.268 & 0.307 to 0.780 & $<0.001^{\star \star \star}$ \\
\hline Length of mechanical ventilation (in acute-care ICU) & 0.008 & -0.115 to 0.131 & 0.896 \\
\hline Length of ICU stay (in acute-care ICU) & 0.086 & -0.035 to 0.209 & 0.163 \\
\hline \multicolumn{4}{|l|}{ Psychological variables at (postacute) ICU } \\
\hline History of depressive disorder & 0.073 & -0.113 to 0.454 & 0.236 \\
\hline History of harmful alcohol consumption & 0.030 & -0.223 to 0.368 & 0.629 \\
\hline History of anxiety disorder & -0.032 & -0.531 to 0.310 & 0.604 \\
\hline
\end{tabular}

Method of univariate logistic regression analysis: Enter.

${ }^{*} \mathrm{P} \leq 0.05,{ }^{* * *} \mathrm{p} \leq 0.001$.

$\dagger \mathrm{t}=5$ missing values.

$\ddagger \mathrm{n}=34$ missing values.

CAM-ICU, Confusion Assessment Method for the Intensive Care Unit.

could be identified, that is, sepsis in postacute ICU, the number of medical comorbidities, the degree of functional impairment as assessed by the Barthel index and a diagnosis of organic brain syndrome (table 2).

\section{Association with fatigue, health-related quality of life, institutionalisation or death at follow-up}

Cronbach's $\alpha$ for the MFI-20 was 0.91 (with a range of 0.50 for 'reduced motivation' and 0.86 for 'mental fatigue') at t2 and 0.93 (with a range of 0.64 for 'reduced motivation' and 0.84 for 'mental fatigue') at t3. A correlation analysis revealed a significantly positive, small-sized association between the CAM-ICU-7 DSS score at the time of recruitment (t1) and mental fatigue at t2 (Spearman's correlation $=0.247, \mathrm{p}=0.008,95 \%$ CI 0.132 to 0.373 ), but not at t3 (Spearman's correlation $=0.029, \mathrm{p}=0.773,95 \% \mathrm{CI}-0.092$ to 0.150 , see online supplemental table S3).

In the present study, Cronbach's $\alpha$ for the health-related quality of life was 0.74 at t2 and 0.77 at t3. No significant associations could be shown for the CAM-ICU-7 DSS with the health-related quality of life.
A worse CAM-ICU DSS was associated with death or institutionalisation in a hospital/nursing home at $\mathrm{t} 2$ but not at t3 (t2: Spearman's correlation $=0.318, \mathrm{p}<0.001$; t3: Spearman's correlation $=0.140, \mathrm{p}=0.125$ ) (see online supplemental table S3).

In multinomial regression analyses, the CAM-ICU-7 DSS at postacute care (t1) could be identified as independent risk factor for both, institutionalisation and death, 3 months following ICU discharge (t2) (see table 3). At t2, an increase of the CAM-ICU-7 score by one point can increase the risk for being institutionalised rather than being discharged home by the factor 5.5 , and the risk of dying by the factor 8.0. At $\mathrm{t} 3$, the increase of the CAMICU DSS was associated with a nearly five-times increased risk of dying (see online supplemental table S4).

\section{DISCUSSION}

In the present study, we investigated the frequency of delirium in CCI patients and found a lower rate of delirium than formerly reported in comparable patient 
Table 3 Sociodemographic and clinical characteristics associated with the outcome (dead, institutionalised vs discharged home) at 3 months follow-up (t2)

\begin{tabular}{|c|c|c|c|c|}
\hline \multirow[b]{2}{*}{ Factors } & \multicolumn{2}{|l|}{ Institutionalised at t2 } & \multicolumn{2}{|l|}{ Death at $\mathrm{t} 2$} \\
\hline & Adjusted OR (95\% Cl) & $\begin{array}{l}P \text { value in } \\
\text { final model }\end{array}$ & Adjusted OR (95\% Cl) & $\begin{array}{l}P \text { value in } \\
\text { final model }\end{array}$ \\
\hline Gender & 2.060 (0.687 to 6.180$)$ & 0.197 & $1.639(0.507$ to 5.303$)$ & 0.409 \\
\hline Family status & 1.724 (0.686 to 4.332$)$ & 0.247 & 0.828 (0.263 to 2.612$)$ & 0.748 \\
\hline Length of mechanical ventilation & $0.435(0.179$ to 1.059$)$ & 0.067 & $1.552(0.474$ to 5.075$)$ & 0.467 \\
\hline Length of ICU stay & 3.878 (1.605 to 9.367$)$ & $0.003^{* *}$ & 0.796 (0.217 to 2.918$)$ & 0.730 \\
\hline Number of sepsis episodes & 0.951 (0.571 to 1.585$)$ & 0.848 & $1.716(1.077$ to 2.735$)$ & $0.023^{\star}$ \\
\hline Number of comorbidities & $0.843(0.496$ to 1.433$)$ & 0.529 & 1.608 (0.893 to 2.894$)$ & 0.113 \\
\hline
\end{tabular}

${ }^{*} \mathrm{P} \leq 0.05,{ }^{* *} \mathrm{p} \leq 0.01$.

CAM-ICU, Confusion Assessment Method for the Intensive Care Unit.

populations. ${ }^{16} 17$ However, we only used one single assessment point in order to evaluate a diagnosis of delirium and DS, instead of using daily assessments over a longer period of time. Similarly, low rates of delirium have been reported in studies using a single assessment point ${ }^{43} 44$ (eg, 9.6\% in elderly emergency department patients ${ }^{43}$; $2.1 \%$ for community hospital patients ${ }^{44}$ ). Above, our rate falls within a wide range of delirium incidences, as previously described for medical inpatients (eg, 3\%-29\%). ${ }^{45}$

The severity of the medical illness, complication by sepsis, a diagnosis of organic brain syndrome and number of comorbidities, were associated with DS during postacute ICU in these patients. This has been already shown in a study by Hope et al. ${ }^{16}$ As underlying mechanism for the association of more medical than sociodemographic and psychological factors influencing the evolvement of severe delirium symptoms, and a delirium as well, a shared pathophysiology comprising neuroinflammation, an aberrant stress response, neurotransmitter imbalances and neuronal network alterations are supposed. ${ }^{46}$ Moreover, a combination of medications, traumatic stress, pain, inflammation, hypoxaemia and brain injury during the prolonged stay on ICU may contribute to more severe delirium symptoms and promote the development of psychiatric disorders. ${ }^{47}$ Thus, appropriate pharmacological and non-pharmacological strategies are warranted in order to improve long-term neurocognitive outcomes and prevent the evolvement of a delirium. These strategies include a cautious use of benzodiazepines, the use of either propofol or dexmedetomidine instead, the short-term use of haloperidol or an atypical antipsychotic in case of agitation or self-harm. Furthermore, an early physical mobilisation, the reduction of sleep deprivation, support with reorientation, application of adequate pain and agitation management protocols and the improvement of hearing as well as vision are essential. ${ }^{46} 48$ In line with this, future research should address the investigation of potential modifiable and also iatrogenic risk factors, such as the reduction of sedation, treatment of hypoxia and of sepsis, in order to decrease worse outcomes in long-term cognitive functioning. ${ }^{49}$

We could not find any association between the duration of mechanical ventilation, and DS at postacute ICU or the MFI-20 subscale 'mental fatigue' at follow-up (see online supplemental table S5). This is in line with findings by Girard et $a l,{ }^{28}$ who also could not find any association. Furthermore, there is evidence that mechanical ventilation does not alter the risk of delirium occurrence. ${ }^{48}$ However, mechanically ventilated patients, especially those with long-term ventilation, are prone to develop acute and long-term forms of cognitive impairments. ${ }^{15} 50$ In contrast, most studies confirmed that delirium or more severe delirium symptoms are rather causes than consequences for a longer hospital length of stay or length of mechanical ventilation, respectively. ${ }^{45} 5152$ In line with this, we also did not find an association between the length of mechanical ventilation/length of ICU stay in acute-care hospital and DSS in the present study population. A further analysis confirmed a significantly positive association between the total length of mechanical ventilation/length of stay (summarising both acute-care and postacute care ICU stay) with the CAM-ICU-7 DSS (online supplemental table S5). The inconsistencies of evidence may be explained by differences in measurement methods. In order to assess the extent of cognitive dysfunctions, we used a simple screening score of the CAM-ICU and the subscale 'mental fatigue' of the MFI-20 questionnaire, instead of using more objective neuropsychological tests. ${ }^{47}$

The present finding of a significant though smallsized positive association between DS at postacute ICU and mental fatigue at $\mathrm{t} 2$ but not $\mathrm{t} 3$ is in line with findings by Girard et al, ${ }^{28}$ showing that long-term cognitive performance can be predicted by the cognitive status 
during ICU stay in survivors of CCI. Another study could show that mechanically ventilated patients who developed delirium during ICU stay were nine times more likely to be discharged with cognitive impairment than were those without delirium. ${ }^{15}$ However, our results should be interpreted cautiously since mental fatigue is rather a subjective measure of the perceived difficulty to sustain task performance. Future studies should therefore validate this subjective measure on more objective instruments such as the Repeatable Battery for the Assessment of Neuropsychological Status and Trail Making Test or a more comprehensive neuropsychological test battery. ${ }^{28}$

In our present study, DS at post-ICU turned out to be an independent risk factor for being institutionalised or dead at follow-up. This is line with former findings in elderly medical inpatients, ${ }^{21}$ general hospital, ${ }^{20}$ ICU patients ${ }^{23}$ and mechanically ventilated patients. ${ }^{15}$ Nevertheless, the present results should be regarded as preliminary and need replication in further studies. Moreover, there was no significant association of the CAM-ICU-7 DSS with health-related quality of life after hospital discharge. This finding also needs replication in further studies.

Our findings should be critically evaluated considering the following methodological shortcomings. First, the present study is a post hoc analysis of a study primarily designed to assess post-traumatic stress disorder in CCI patients following prolonged treatment on ICU. ${ }^{34}$ The assessment of the CAM-ICU was originally applied in order to ascertain that the patients are cognitively able to be interviewed according to assess an acute/posttraumatic stress disorder. Patients who were cognitively impaired, for instance, showing a positive screening of the CAM-ICU subscales 'attention' and 'disorganised thinking' or presented a delirium, were screened again after 1 week. In case the delirium or delirium symptoms persisted, the patients were excluded from further study participation. Thus, patients with more severe delirium symptoms or persistent delirium dropped out, leading to a selection bias-towards patients without a full or subsyndromal delirium manifestation or with only mild delirium symptoms.

Additionally, the CAM-ICU was not assessed at multiple time points (eg, daily, biweekly) during the ICU stay, but only twice at maximum. However, a single CAM-ICU assessment in patients who have a prolonged ICU stay provides a less reliable estimate of delirium frequency. Future studies should apply the CAM-ICU more often, for example, daily ${ }^{28}$ or biweekly, ${ }^{16}$ in order to gain access to the number of days spent in delirium. The latter has been found to be an independent predictor of long-term survival. ${ }^{15} 5354$

Furthermore, we did not have any information regarding further relevant variables measured before the patients were transferred to the chronic care facility, including prehospital functional ability, pre-existing cognitive function or frailty. These variables display major influencing factors for long-term brain dysfunctions. Future studies should therefore address for example, activities of daily living or a functional independence measure by interviewing a family member or surrogate. Moreover, future research should apply different tools in order to assess delirium and delirium symptom severity (eg, Delirium Rating Scale-Revised-98). This would allow to specify the convergent validity of the CAM-ICU-7 DSS.

Altogether, the present study underlines the urgency for ongoing clinical and research efforts to reduce cognitive and thus functional disability in older adults surviving serious medical illnesses ${ }^{55} 56$ and to provide appropriate multidisciplinary resources to facilitate both, physical and neuropsychological rehabilitation. Consequently, adequate delirium management strategies as well as early mobility and prevention programmes are needed in order to mitigate cognitive and physical long-term sequelae as well as high societal costs of chronical critical illness. ${ }^{28}$

\section{CONCLUSION}

In conclusion, nearly every 10th CCI patient showed severe or most severe delirium symptoms. Primarily clinical factors have been identified to be associated with DS during the postacute ICU stay in these patients, including a diagnosis of sepsis, number of medical comorbidities and the extent of functional impairment. Most importantly, patients showing a higher DSS during their stay on the weaning ward, also suffered from more severe mental fatigue and had an increased risk for being institutionalised or dead at follow-up. Thus, a single assessment of a DS sum score during admission at postacute ICU can be used to assess the clinical course in CCI patients. Future research should focus on interventions designed to improve the management of delirium and severe delirium symptoms in CCI patients.

\section{Author affiliations}

${ }^{1}$ Department of Psychotherapy and Psychosomatic Medicine, Medizinische Fakultät Carl Gustav Carus, Technische Universität Dresden, Dresden, Sachsen, Germany ${ }^{2}$ Institute of Psychosocial Medicine, Psychotherapy and Psychooncology, Jena University Hospital, Jena, Thüringen, Germany

${ }^{3}$ Center for Sepsis Control and Care, Jena University Hospital, Jena, Thüringen, Germany

Contributors G-BW and JR were principal investigators of the present study, were responsible for data analysis and preparation of the present manuscript. KW and BS gave advice according to study conduction. Furthermore, they provided technical and personal resources for data collection and proof-read the manuscript.

Funding The present study was supported by the German Federal Ministry of Education and Research grant $01 \mathrm{E} 01002$.

Competing interests None declared.

Patient consent for publication Not required.

Ethics approval The study was conducted in accordance with the Declaration of Helsinki and approved by the Ethics Committee of the Friedrich-Schiller University, Jena, Germany (No. 3278-10/11). All patients or a representative signed in written informed consent.

Provenance and peer review Not commissioned; externally peer reviewed. 
Data availability statement Data are available on reasonable request. The data are available on reasonable request by contacting the first author of the present study (Gloria.Wintermann@uniklinikum-dresden.de). Before reuse of the patient data, a deidentification is necessary.

Supplemental material This content has been supplied by the author(s). It has not been vetted by BMJ Publishing Group Limited (BMJ) and may not have been peer-reviewed. Any opinions or recommendations discussed are solely those of the author(s) and are not endorsed by BMJ. BMJ disclaims all liability and responsibility arising from any reliance placed on the content. Where the content includes any translated material, BMJ does not warrant the accuracy and reliability of the translations (including but not limited to local regulations, clinical guidelines, terminology, drug names and drug dosages), and is not responsible for any error and/or omissions arising from translation and adaptation or otherwise.

Open access This is an open access article distributed in accordance with the Creative Commons Attribution Non Commercial (CC BY-NC 4.0) license, which permits others to distribute, remix, adapt, build upon this work non-commercially, and license their derivative works on different terms, provided the original work is properly cited, appropriate credit is given, any changes made indicated, and the use is non-commercial. See: http://creativecommons.org/licenses/by-nc/4.0/.

\section{ORCID iDs}

Gloria-Beatrice Wintermann http://orcid.org/0000-0002-6292-0852

Jenny Rosendahl http://orcid.org/0000-0001-7535-7571

\section{REFERENCES}

1 Nelson JE, Cox CE, Hope AA, et al. Chronic critical illness. Am J Respir Crit Care Med 2010;182:446-54

2 Zink W, Kollmar R, Schwab S. Critical illness polyneuropathy and myopathy in the intensive care unit. Nat Rev Neurol 2009;5:372-9.

3 Angus DC, van der Poll T. Severe sepsis and septic shock. N Engl J Med 2013;369:840-51

4 Iwashyna TJ, Hodgson CL, Pilcher D, et al. Towards defining persistent critical illness and other varieties of chronic critical illness. Crit Care Resusc 2015;17:215-8.

5 Zhou C, Wu L, Ni F, et al. Critical illness polyneuropathy and myopathy: a systematic review. Neural Regen Res 2014;9:101-10.

6 Tepper M, Rakic S, Haas JA, et al. Incidence and onset of critical illness polyneuropathy in patients with septic shock. Neth J Med 2000;56:211-4

7 Iwashyna TJ, Cooke CR, Wunsch $\mathrm{H}$, et al. Population burden of long-term survivorship after severe sepsis in older Americans. J Am Geriatr Soc 2012;60:1070-7.

8 Iwashyna TJ, Ely EW, Langa KM. Incident severe sepsis is associated with substantial long-term cognitive and functional decline: 8-year follow-up of the health and retirement study. Am J Resp Crit Care 2010;181.

9 Iwashyna TJ, Ely EW, Smith DM, et al. Long-Term cognitive impairment and functional disability among survivors of severe sepsis. JAMA 2010;304:1787-94.

10 DeForest A, Blinderman CD. Persistent delirium in chronic critical illness as a prodrome syndrome before death. $J$ Palliat Med 2017;20:569-72.

11 Inouye SK, van Dyck CH, Alessi CA, et al. Clarifying confusion: the confusion assessment method. A new method for detection of delirium. Ann Intern Med 1990;113:941-8.

12 McCoy TH, Hart KL, Perlis RH. Characterizing and predicting rates of delirium across General Hospital settings. Gen Hosp Psychiatry 2017;46:1-6.

13 Ryan DJ, O'Regan NA, Caoimh Ronán Ó, et al. Delirium in an adult acute hospital population: predictors, prevalence and detection. BMJ Open 2013;3:e001772.

14 Inouye SK, Westendorp RGJ, Saczynski JS. Delirium in elderly people. Lancet 2014;383:911-22.

15 Ely EW, Shintani A, Truman B, et al. Delirium as a predictor of mortality in mechanically ventilated patients in the intensive care unit. JAMA 2004;291:1753-62.

16 Hope AA, Morrison RS, Du Q, et al. Risk factors for long-term brain dysfunction after chronic critical illness. Ann Am Thorac Soc 2013;10:315-23.

17 Nelson JE, Tandon N, Mercado AF, et al. Brain dysfunction: another burden for the chronically critically ill. Arch Intern Med 2006;166:1993-9.

18 Ely EW, Inouye SK, Bernard GR, et al. Delirium in mechanically ventilated patients: validity and reliability of the confusion assessment method for the intensive care unit (CAM-ICU). JAMA 2001;286:2703-10.

19 Gusmao-Flores D, Salluh JIF, Chalhub Ricardo Ávila, et al. The confusion assessment method for the intensive care unit (CAMICU) and intensive care delirium screening checklist (ICDSC) for the diagnosis of delirium: a systematic review and meta-analysis of clinical studies. Crit Care 2012;16:R115.

20 Inouye SK, Kosar CM, Tommet D, et al. The CAM-S: development and validation of a new scoring system for delirium severity in 2 cohorts. Ann Intern Med 2014;160:526-33.

21 Cole M, McCusker J, Dendukuri N, et al. The prognostic significance of subsyndromal delirium in elderly medical inpatients. J Am Geriatr Soc 2003;51:754-60.

22 Serafim RB, Soares M, Bozza FA, et al. Outcomes of subsyndromal delirium in ICU: a systematic review and meta-analysis. Crit Care 2017;21:179.

23 Khan BA, Perkins AJ, Gao S, et al. The confusion assessment method for the ICU-7 delirium severity scale: a novel delirium severity instrument for use in the ICU. Crit Care Med 2017;45:851-7.

24 Van Rompaey B, Schuurmans MJ, Shortridge-Baggett LM, et al. Risk factors for intensive care delirium: a systematic review. Intensive Crit Care Nurs 2008;24:98-107.

25 van den Boogaard M, Pickkers P, Slooter AJC, et al. Development and validation of PRE-DELIRIC (prediction of delirium in ICU patients) delirium prediction model for intensive care patients: observational multicentre study. BMJ 2012;344:e420.

26 van den Boogaard M, Schoonhoven L, Maseda E, et al. Recalibration of the delirium prediction model for ICU patients (PRE-DELIRIC): a multinational observational study. Intensive Care Med 2014;40:361-9.

27 Linkaite G, Riauka M, Bunevičiūtė I, et al. Evaluation of PRE-DELIRIC (prediction of delirium in ICU patients) delirium prediction model for the patients in the intensive care unit. Acta Med Litu 2018;25:14-22.

28 Girard TD, Jackson JC, Pandharipande PP, et al. Delirium as a predictor of long-term cognitive impairment in survivors of critical illness. Crit Care Med 2010;38:1513-20.

29 Thomason JWW, Shintani A, Peterson JF, et al. Intensive care unit delirium is an independent predictor of longer hospital stay: a prospective analysis of 261 non-ventilated patients. Crit Care 2005;9:R375-81.

30 Fong TG, Jones RN, Shi P, et al. Delirium accelerates cognitive decline in Alzheimer disease. Neurology 2009;72:1570-5.

31 Davydow DS, Gifford JM, Desai SV, et al. Posttraumatic stress disorder in general intensive care unit survivors: a systematic review. Gen Hosp Psychiatry 2008;30:421-34.

32 Davydow DS, Hough CL, Langa KM, et al. Depressive symptoms in spouses of older patients with severe sepsis. Crit Care Med 2012;40:2335-41.

33 Wintermann G-B, Rosendahl J, Weidner K, et al. Fatigue in chronically critically ill patients following intensive care - reliability and validity of the multidimensional fatigue inventory (MFI-20). Health Qual Life Outcomes 2018;16:37.

34 Wintermann G-B, Brunkhorst FM, Petrowski K, et al. Stress disorders following prolonged critical illness in survivors of severe sepsis. Crit Care Med 2015;43:1213-22.

35 Klugkist M, Sedemund-Adib B, Schmidtke C, et al. [Confusion Assessment Method for the Intensive Care Unit (CAM-ICU): diagnosis of postoperative delirium in cardiac surgery]. Anaesthesist 2008;57:464-74.

36 Rollnik JD. The early rehabilitation Barthel index (ERBI). Rehabilitation 2011;50:408-11.

37 O'Sullivan SB, Schmitz TJ. Physical rehabilitation assessment and treatment. Philadelphia: F.A. Davis Company, 2006.

38 Sessler CN, Gosnell MS, Grap MJ, et al. The Richmond AgitationSedation scale: validity and reliability in adult intensive care unit patients. Am J Respir Crit Care Med 2002;166:1338-44.

39 Smets EM, Garssen B, Bonke B, et al. The multidimensional fatigue inventory (MFI) psychometric qualities of an instrument to assess fatigue. J Psychosom Res 1995;39:315-25.

40 Schwarz R, Krauss O, Hinz A. Fatigue in the general population. Onkologie 2003;26:140-4.

41 Rabin R, de Charro F. EQ-5D: a measure of health status from the EuroQol group. Ann Med 2001;33:337-43.

42 Hinz A, Brähler E, Schwarz R, et al. [How useful is the calculation of total scores for questionnaires concerning health related quality of life?]. Psychother Psychosom Med Psychol 2005;55:221-8.

43 Elie M, Rousseau F, Cole M, et al. Prevalence and detection of delirium in elderly emergency department patients. CMAJ 2000;163:977-81.

44 McCoy TH, Snapper L, Stern TA, et al. Underreporting of delirium in statewide claims data: implications for clinical care and predictive modeling. Psychosomatics 2016;57:480-8. 
45 Siddiqi N, House AO, Holmes JD. Occurrence and outcome of delirium in medical in-patients: a systematic literature review. Age Ageing 2006;35:350-64.

46 Slooter AJC, Van De Leur RR, Zaal IJ. Delirium in critically ill patients. Handb Clin Neurol 2017;141:449-66.

47 Hopkins RO, Key CW, Suchyta MR, et al. Risk factors for depression and anxiety in survivors of acute respiratory distress syndrome. Gen Hosp Psychiatry 2010;32:147-55.

48 Devlin JW, Skrobik Y, Gélinas C, et al. Clinical practice guidelines for the prevention and management of pain, Agitation/Sedation, delirium, immobility, and sleep disruption in adult patients in the ICU. Crit Care Med 2018:46:e825-73.

49 Girard TD, Thompson JL, Pandharipande PP, et al. Clinical phenotypes of delirium during critical illness and severity of subsequent long-term cognitive impairment: a prospective cohort study. Lancet Respir Med 2018;6:213-22.

50 Pandharipande P, Cotton BA, Shintani A, et al. Prevalence and risk factors for development of delirium in surgical and trauma intensive care unit patients. J Trauma 2008;65:34-41.
51 Émond M, Boucher V, Carmichael P-H, et al. Incidence of delirium in the Canadian emergency department and its consequences on hospital length of stay: a prospective observational multicentre cohort study. BMJ Open 2018;8:e018190.

52 Tilouche N, Hassen MF, Ali HBS, et al. Delirium in the intensive care unit: incidence, risk factors, and impact on outcome. Indian J Crit Care Med 2018;22:144-9.

53 Pandharipande PP, Girard TD, Jackson JC, et al. LongTerm cognitive impairment after critical illness. N Engl J Med 2013;369:1306-16.

54 Pisani MA, Kong SYJ, Kasl SV, et al. Days of delirium are associated with 1-year mortality in an older intensive care unit population. $A m \mathrm{~J}$ Respir Crit Care Med 2009;180:1092-7.

55 Schweickert WD, Pohlman MC, Pohlman AS, et al. Early physical and occupational therapy in mechanically ventilated, critically ill patients: a randomised controlled trial. Lancet 2009;373:1874-82.

56 Needham DM, Korupolu R, Zanni JM, et al. Early physical medicine and rehabilitation for patients with acute respiratory failure: a quality improvement project. Arch Phys Med Rehabil 2010;91:536-42. 